\title{
Article
}

\section{Multidevice Map-Constrained Fingerprint- Based Indoor Positioning Using 3-D Ray Tracing}

\author{
Raspopoulos, Marios
}

Available at http://clok.uclan.ac.uk/21759/

Raspopoulos, Marios ORCID: 0000-0003-1513-6018 (2018) Multidevice MapConstrained Fingerprint-Based Indoor Positioning Using 3-D Ray Tracing. IEEE Transactions on Instrumentation and Measurement, 67 (2). pp. 466-476. ISSN $0018-9456$

It is advisable to refer to the publisher's version if you intend to cite from the work.

http://dx.doi.org/10.1109/TIM.2017.2774181

For more information about UCLan's research in this area go to http://www.uclan.ac.uk/researchgroups/ and search for <name of research Group>.

For information about Research generally at UCLan please go to http://www.uclan.ac.uk/research/

All outputs in CLoK are protected by Intellectual Property Rights law, including Copyright law. Copyright, IPR and Moral Rights for the works on this site are retained by the individual authors and/or other copyright owners. Terms and conditions for use of this material are defined in the policies page.

\section{CLoK}

Central Lancashire online Knowledge www.clok.uclan.ac.uk

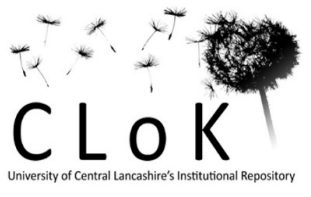




\title{
Multi-device Map-constrained Fingerprint-based Indoor Positioning using 3D Ray Tracing
}

\author{
Marios Raspopoulos
}

\begin{abstract}
This paper studies the use of deterministic channel modelling through 3D Ray Tracing (RT) for constructing deviceindependent radiomaps for Wi-Fi RSSI-based fingerprinting indoor positioning, applicable to different devices. Device heterogeneity constitutes a limitation in fingerprint-based approaches and also constructing radiomaps through extensive in-situ measurement campaigns is laborious and time-consuming even with a single device let alone the need for radiomaps constructed using multiple different devices. This work tackles both challenges through the use of 3D RT for radiomap generation in conjunction with data calibration using a small set of device-specific measurements to make the radiomap device-independent. The efficiency of this approach is evaluated using simulations and measurements in terms of the time spent to generate the radiomap, the amount of device-specific data required for calibration and in terms of the achievable positioning accuracy. Potential accuracy improvements in the RT-based indoor positioning processes are further investigated, by studying the use of map constraints into the algorithm in the form of a-priori probabilities. In this approach, a Route Probability Factor (RPF), which reflects the likelihood of a user being in various locations inside the environment is used. The outcome of the evaluation process which includes a study of different RPF distributions, indicates the validity of the approach, demonstrated by a reduction in the positioning error for various devices. The versatility of this approach is also demonstrated for different scenarios, different devices and by considering different device-handling conditions.
\end{abstract}

Index Terms-Indoor Positioning, Fingerprinting, MapConstrains, Ray Tracing, Wi-Fi, RSSI, Route Probability Factor.

\section{INTRODUCTION}

$\mathbf{T}$ HE explosive growth of mobile communications and the Internet of Things (IoT) has come along with the emergence of Location-Based Services (LBS) which find applications in various fields such as social networks, entertainment, security, health and rescue services and many others. While the positioning problem was solved for outdoor environments through the use of Global Navigation Satellite Systems (GNSS), Indoor Positioning Systems (IPS) still remains a big challenge and an active area of research [1]. OpusResearch in a 2014 report [2], predict that by 2018, roughly $\$ 10$ billion in consumer spending will be influenced by indoor location. This is also supported by a report by IndoorAtlas [3] which presents the results of a 2016 market research on the indoor positioning market indicating that the budget spent on IPS will be increased by $3.07 \%$ in the next five years. The reason for

M. Raspopoulos was with the University of Central Lancashire, e-mail: mraspopoulos@uclan.ac.uk

Manuscript received June 8, 2017; revised August 18, 2017; accepted September 23, 2017. this is that it is estimated that today, more than $80-90 \%$ of people's time is spent indoors. Possibly the most important finding is that almost all $(98 \%)$ respondents state that subtwo-meter accuracy is important for most of these indoor applications. This means that improving the accuracy using off-the-shelf technologies is of paramount importance and this drives our urge to further pursue research in this area.

While various approaches which achieve sub-meter accuracy have been proposed in literature, these usually require technologies which have not yet been integrated in the latest Wi-Fi standards. Such techniques include Ultra-Wideband (UWB) [4]-[6] or Magnetic Field Intensity (MFI) [7], [8] technologies etc. Because of this, fingerprint-based positioning [9]-[11] which became a popular topic of research over the last decades, still remains an active area of research mainly because of its simplicity and its potential to be applicable using off-the-shelf technologies. Fingerprinting, consists of an off-line phase where pre-measured location-dependent signal parameters (e.g. RSSI), known as "fingerprints" are stored in a database (radiomap) and an on-line phase where the realtime measurement is correlated with the fingerprints in the radiomap to estimate the position.

Generating and maintaining the radiomap is crucial and can be achieved either through extensive in-situ measurements or through channel modelling. Measurements may lead to more accurate fingerprints, but the process is laborious and timeconsuming and a big challenge is that the applicability of the measured radiomap is reduced if the wireless environment is altered. Channel modelling techniques, such as Ray Tracing (RT) have been proposed for the generation of the radiomap [9], [12]-[14]. Still, RT accuracy is subject to the precise definition of the geometry [15] and morphology [16] of the environment and also the accurate definition of the antennas and other parameters. Such information is usually hard to obtain and this might necessitate the crude calibration of the RT tool in order to achieve higher accuracy.

A basic limitation of fingerprinting techniques is that device heterogeneity may degrade the positioning performance when the type of device to be positioned is different from the one that was used to generate the radiomap. This heterogeneity is based on the differences that may arise due to the different antenna characteristics of the wireless device which are usually difficult to know or predict. There is work reported that tries to address this; mainly by calibrating the RSSI measurements manually collected using the device to be positioned to better match the fingerprints contained in the radiomap (possibly created using another device), either by lineary transforming the collected measurements [17], [18], or by recording the 
signal ratios between pairs of Access Points [19].

This work proposes a process for the generation of a unique fingerprint radiomap using 3D RT and the estimation of a set of linear-transformation parameters through data-fitting of a small set of manually-collected device-specific fingerprints to this radiomap to make the process device-independent. The focus is on the amount of fingerprints that need to be manually collected using each device in order to obtain appropriate linear transformation parameters that guarantee a low positioning error.

Many attempts were focused in literature on improving the accuracy of fingerprinting positioning by imposing and/or fusing additional (to the radio) context collected by the device sensors. The accuracy seems to depend on the volume and quality of this context and it was proven that the more information included in the process the higher the probability for producing more accurate position estimates [20]-[22]. This information may include radio parameters such as Received Signal Strength Indication (RSSI) [23], Magnetic Field Intensity (MFI) [7] and Channel Impulse Responses (CIR) [24] which could also be cleverly combined or fused to improve the performance [25]. However, the sole dependence on radio parameters imposes various limits that are hard to overcome therefore attempts where diverted in the use of non-radio parameters such as inertial, temperature, light/illumination measurements etc. Even though modern mobile devices are equipped with a variety of sensors, different devices may contain different ones, something which makes the process difficult to generalize or standardize. Therefore this paper proposes the utilisation of the available environment map description to aid the localisation process. By doing so, the expected mobility of the user is expected to be constrained and different probabilities could be assigned to different areas of the environment where the user is likely to be.

This paper combines the author's previous works on crossdevice [26] and map-aided [27] fingerprinting into a unified solution capable of achieving the sub-two-meter accuracy requirement reported in [3]. The rest of the paper is organized as follows: In Section III the related background work is briefly summarized. Section [III describes the test environment and measurement methodology to generate device-specific radiomaps and Section IV] describes the proposed approach for the generation of the RT radiomap. The performance evaluation of the multi-device fingerprint approach and the further improvements through the use of map-constrains are presented in sections $\nabla$ and $\mathrm{VI}$ respectively. The results are compared to other methods in literature in section VII

\section{BACKGROUND}

\section{A. Propagation Modelling}

Ray Tracing (RT) is considered as the dominant deterministic modelling approach for predicting radio propagation in wireless communications. It is based on Geometrical Optics (GO) where for a a given detailed geometric and morphological description of the environment, together with the specifications of the antennas, identifies all the possible ray paths and then applies electromagnetic theory to calculate the total received power.
Channel modeling has been proposed for radiomap creation for fingerprint-based positioning [9], [12], [28]. The authors of [14] have used 3D RT and compared its performance and computational cost against the previously reported methods for indoor environments achieving a mean positioning error of $2.3 m$ (using the same device) and also examined the sensitivity on the accuracy due to the inaccurate material parameters defined in the RT tool. The aforementioned works use RT for RSSI-based fingerprinting, but the authors of [24] have used RT to assist the creation of radiomaps for their fingerprintbased solution which uses a fused combination of RSSI and the Channel Impulse Response (CIR) as the position signature (fingerprint). Also, in [29] 3D RT has been used to create radiomaps for device-based and device-free cases achieving a mean error of $1.6 \mathrm{~m}$ and $2.44 \mathrm{~m}$ respectively, however this has done for a simple Line of Sight (LoS) scenario which is a limited case in indoor environments. This work proposes the use of RT to generate fingerprint-based radiomaps for both Non-LoS and LoS which can be calibrated, transformed and accurately be used with different types devices.

\section{B. Fingerprint-based Positioning Algorithms}

Fingerprint-based positioning is classified into deterministic and probabilistic approaches. Deterministic approaches estimate the position as a weighted convex combination of the reference locations [30]. Usually, the $K$ reference locations with the shortest distance (usually the Euclidean [31]) $D_{i}$, between the measured fingerprint $s$ and the fingerprints $r_{i}=\left[r_{i 1}, \ldots, r_{i N}\right]^{T}$ in the radiomap and then estimating the location $\hat{\ell}$ by calculating a weighted average of the locations that correspond to the $K$ nearest matches:

$$
\widehat{\ell}=\sum_{i=1}^{K}\left(\frac{w_{i}}{\sum_{j=1}^{K} w_{j}} \ell_{i}^{\prime}\right), \quad D_{i}=\sqrt{\sum_{j=1}^{N}\left(r_{i j}-s_{j}\right)^{2}}
$$

,where $r_{i j}$ and $s_{j}$ denote the RSSI values related to the $j$-th Access Point (AP) $(j=1, \ldots, N)$ for the radiomap fingerprint and the on-line observed measurement respectively. Weights $w_{i}$ are estimated using the inverse of the Euclidean distance $D_{i}$ [32]. The $K$-Nearest Neighbour (KNN) method assumes equal weights $w_{i}$ for the $K$ candidate reference locations, while setting $K=1$ leads to the simple NN method [31], [33], [34]. In probabilistic methods, the location is estimated by maximising a conditional posterior probability $p\left(\ell_{i} \mid s\right)$ given an observed fingerprint $s$, a fingerprint database and a prior probability $p\left(\ell_{i}\right)$ which describes the probability of the target being in a specific loacation [35], [36].

The proposed solution combines the probabilistic with the deterministic approach by using the prior $p\left(\ell_{i}\right)$ into the WKNN equation. This probability reflects the likelihood of users being located in specific areas and can be extracted from the environment map considering the position of the furniture and the obstructions or statistically by continuously monitoring the users' movement and constructing frequent route paths. In this context, equation 1 becomes:

$$
\widehat{\ell}=\sum_{i=1}^{K} p\left(\ell_{i}\right)\left(\frac{w_{i}}{\sum_{j=1}^{K} w_{j}} \ell_{i}^{\prime}\right) .
$$




\section{Device Heterogeneity in Fingerprint Positioning}

There is work reported in literature that attempts to tackle the issue of having different types of devives in the fingerprinting positining process. Haeberlen et al. [17] proposed the use of linear mapping for transforming signal strength samples from one device to match the ones of another device, however Park et al. [18] claim that linear transformation alone does not solve the problem and therefore they combine it with a Kernel estimation using a wide Kernel width to further reduce the positioning error. In [37], Vaupel et al. carry out anechoic chamber measurements to obtain the signal strength offsets between different devices and then apply those offsets to calibrate the radiomap according to the device used. This assumes the availability of an anechoic chamber and it limits the applicability of this approach. Kjærgaard in [19] presents a solution where fingerprints are recorded as signal strength ratios between pairs of access points, instead of collecting the absolute readings from each one. The advantage is that this method does not require any device calibration.

All these methods require the collection of a complete radiomap that covers the entire area of interest with at least one device which is used as reference for positioning other devices. Thus, a considerable time and effort for data collection are still required and this grows rapidly if the positioning system is to be deployed in larger-scale setups. The approach in this work addresses this issue efficiently by exploiting an automatically generated RT radiomap that covers all target areas and is applicable for multiple different devices.

\section{Map Information Extraction}

Information extracted from or with the help of maps has been utilized in indoor positioning. For instance, Liao et al. in [38] and Evennou et al. in [39] proposed the use of particle filters to make use of the inherent structure of indoor environments. In order to simplify the calculation complexity of these filters, they suggested the estimation of the locations of people on the environment Voronoi Graph.

In this work, instead of adopting the Voronoi Graphs and the particle filter approach, the most frequent -or most probableroutes are defined, based on observations and by considering the obstructions in the environment map. It is expected that this frequent route would constrain the user motion in areas that (s)he usually resides in. The recording of these routes are based on simple observations and can be achieved by supervised or unsupervised learning techniques. In this work, a supervised approach is adopted as the constrains are manually set in order to prove the principle.

\section{Test EnVironment And Methodology}

To assess the use of RT-generated radiomaps for crossdevice fingerprinting positioning, Wi-Fi RSSI measurements have been collected in an indoor environment using 3 Android devices (HTC Desire HD, Samsung Nexus S and Samsung Galaxy Tab) and 1 laptop computer (Lenovo X100e). Measurements were performed simultaneously with all 4 devices logging data from 6 D-Link 802.11g APs installed inside the building at 110 equally-spaced $(1 \mathrm{~m})$ training locations. At every location 30 samples were recorded $(1 \mathrm{sample} / \mathrm{sec})$ and the mean value, averaged for each AP, has been computed in every location to build each device-specific radiomap. The whole data collection process took 2 hours to complete. For testing purposes, additional RSSI fingerprints have been collected with all devices along a route that comprises of 40 distinct locations, while 10 samples were measured at every test location with no averaging. The test environment is shown in Figure 1. To enable random selection of data which are distributed uniformly in the environment, the area is divided in 7 regions $A_{i}, i=1, \ldots, 7$ representing rooms and large open spaces (see Figure 11, i.e. $\left\{A_{1}: \ell_{j}, j=1, \ldots, 11\right\}$, $\left\{A_{2}: \ell_{j}, j=12, \ldots, 30\right\},\left\{A_{3}: \ell_{j}, j=31, \ldots, 40\right\}$, $\left\{A_{4}: \ell_{j}, j=41, \ldots, 59\right\},\left\{A_{5}: \ell_{j}=60, \ldots, 69\right\}$, $\left\{A_{6}: \ell_{j}, j=70, \ldots, 89\right\},\left\{A_{7}: \ell_{j}, j=90, \ldots, 110\right\}$ where $j$ is the training location index. Each of the six regions contains fingerprints which are in Line of Sight with only one access point whereas the $7^{\text {th }}$ region $\left(A_{1}\right)$ contains fingerprints which do not have a line of sight to any of the access points.

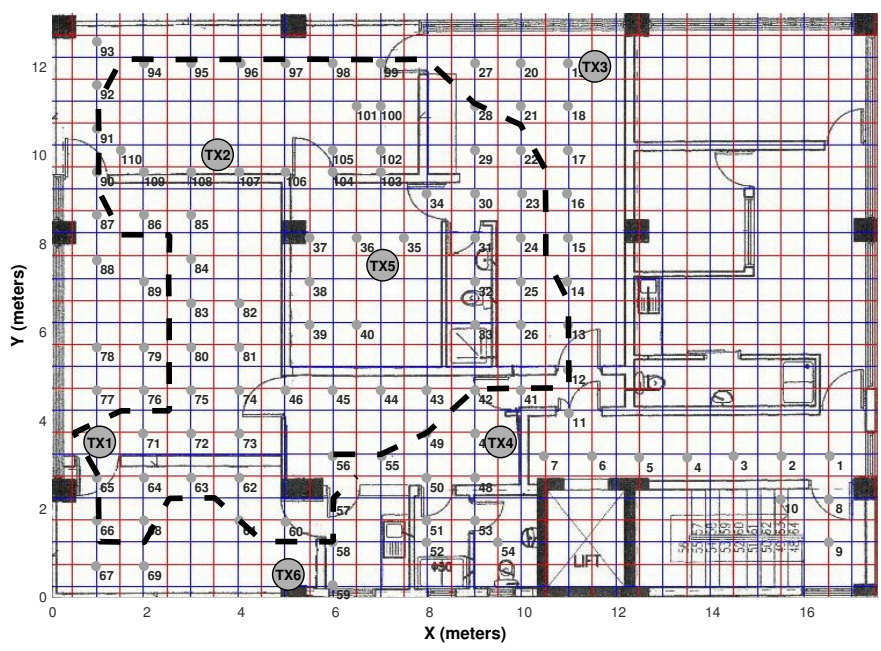

Fig. 1. Experimentation Area Floor Plan (Reference Locations and APs)

\section{Proposed APPROACH}

\section{A. Ray Tracing Radiomap}

For the creation of the fingerprint radiomap, TruNET Wireless [40] was used. It is a 3D deterministic RT propagation simulator which utilises the 3D EM formulation of reflection, refraction and diffraction based on the Uniform Theory of Diffraction [41]. The environment shown in Figure 1 was modelled in the RT Simulator including clutter such as desks, tables etc. and the 3D model is shown in Figure 2, The RT simulation setup consists of 110 receivers defined at the same locations where the measurements were performed. As fast fading effects are typically observed up to half-wavelength distances and since the wavelength of transmission in this experiment is $12.5 \mathrm{~cm}$, at each receiving location a rectangular grid of 36 equally-spaced $(5 \mathrm{~cm})$ isotropic receivers (at $90 \mathrm{~cm}$ height) has been defined to remove any fast fading effects by calculating their local average. The $6 \times 6$ grid was selected to ensure a total size of a $30 \mathrm{~cm} \times 30 \mathrm{~cm}$ rectangular area 
which is comparable to the size of the largest device under test. The 6 APs (at $2.3 m$ heigh) use an omni-directional antenna but since they are mounted on the walls, their pattern is most likely distorted. Therefore, time-gated impulse response measurements have been carried out around a typical omnidirectional antenna placed on the wall, using a Rohde \& Schwarz FSH8 Vector Network Analyser (VNA) in order to better characterise its pattern. The modified antenna pattern has then been imported into TruNET Wireless. Typical values for the electrical parameters [42] were used in order to characterise the building walls and geometric features. These parameters have been further tuned in order to better match the measurements collected with the HTC device. This latter process is known as crude Ray Tracing calibration [43]. The Ray Tracing simulations (compared to the measurements) have achieved a mean error of $5.62 \mathrm{~dB}$ with a standard deviation of $4.23 d B$ using all the measured locations. This error is mainly due to the uncertainties about the exact values of the electrical parameters, the exact radiation pattern of the transmitting antennas, the environment clutter and the uncertainty about the receiver antenna pattern which was assumed isotropic. Since the objective is to devise a methodology where someone will have to collect as few measurements as possible, the RT accuracy using part of the measured data was evaluated. Specifically, 100 combinations of 4 locations were randomly selected per region as described in Section III and similar behaviour was achieved (mean error is $5.6 \pm 0.3 d B$ and the standard deviation is $4.3 \pm 0.2 d B$ ). This means that with only few measurements, a reasonably good fine-tuning of the RT Simulator could be achieved.

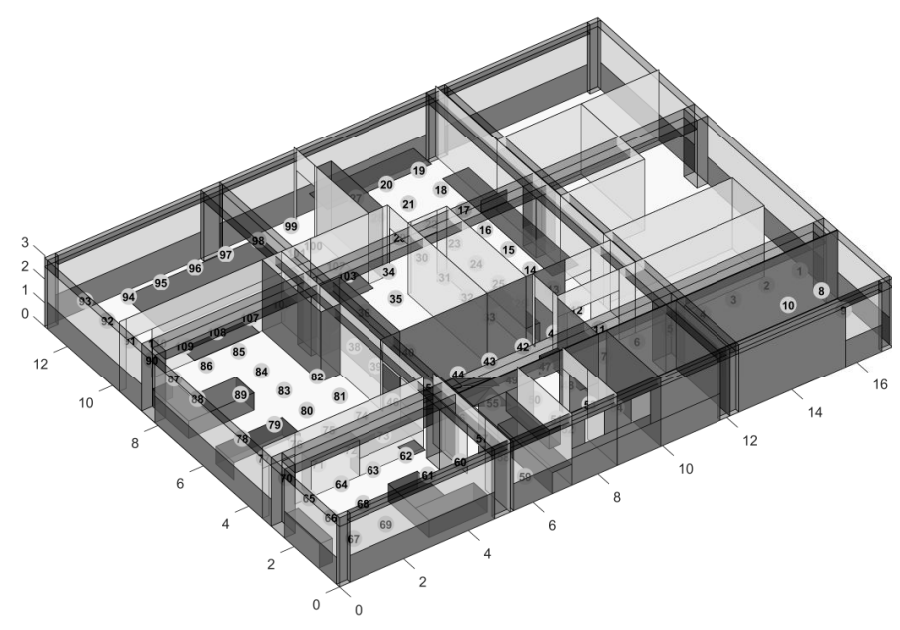

Fig. 2. 3D Model of the Indoor Environment for Ray Tracing Simulations

\section{B. Device Calibration}

The RT-generated radiomap can be used as a reference for multi- and cross-device positioning. The problem is that the devices report the measured RSSI values differently depending on their Wi-Fi adapter hardware and their antenna characteristics (sensitivity and pattern). Therefore, the range of RSSI values can greatly vary among devices, thus rendering the direct use of a single reference radiomap questionable.
Therefore, calibrating the device measurements to match the fingerprints in the radiomap is necessary to deliver a consistent level of performance, regardless of the device used. To increase the applicability of the proposed approach, the use of only a small set of training data for performing this calibration is investigated since the objective is to minimise the time and effort during the off-line phase.

In this work, linear transformation is used in a similar fashion as in [17] by using the training data for mapping the RSSI values recorded with each target device to the RT radiomap, which contains a vector with the mean RSSI values of each AP at every training location. For this reason, linear data fitting is performed in a least-squares sense using the mean RSSI values of each device averaged over multiple fingerprint samples collected at each location. In this fashion, the two linear coefficients are estimated and can be used in a pre-processing step prior to the on-line positioning phase in order to scale the RT radiomap to better correspond to the type of the device to be positioned.

The data fitting is plotted for all 4 devices in Figure 3 indicating a strong linear correlation between the mean RSSI values of each device and the RT radiomap.The linear transformation parameters $(\alpha, \beta)$ are also indicated. It is observed that the Lenovo laptop behaves differently compared to the 3 Android devices and exhibits a large number of values ranging from $-50 \mathrm{dBm}$ to $-45 \mathrm{dBm}$ that correspond to values between $-65 \mathrm{dBm}$ and $-35 \mathrm{dBm}$ with respect to the RT data; see Figure $3 \mathrm{~d}$. This is the reason for the hard limit appearing in the figure. For this specific range of RSSI values a higher order fitting could be applied to address this non-linearity. As it will be shown later in the performance evaluation (Section $\nabla$ ) this behaviour leads to larger positioning error if the RT radiomap is used to localize the X100e device without any calibration.

The linear fitting obtained by using only $\sim 10 \%$ of the training data (dashed line in Figure 3), (the mean RSSI values in the fingerprints from 2 randomly selected locations in each of the 7 regions), is very close to the respective fitting when all the training data is considered (solid line). This indicates that only few data could be used for the device calibration and considerably reduce the measurements time for all devices.

\section{Multi-DeVice Fingerprinting}

An evaluation of the effectiveness of the proposed crossdevice approach is performed with regards to the positioning accuracy compared with the case of using device-specific radiomaps manually collected with each device using the experimental data from the four devices as detailed in Section III

At this point, the focus is on the improvement achieved solely by combining the RT radiomap with the device calibration (data fitting and linear transformation), rather than the fingerprint-based positioning method itself. However, the results are obtained using the well-known Weighted $K$-Nearest Neighbour (WKNN) method for an optimum value of $K=4$ as shown in Figure 4 This value was optimized using the device-specific radiomaps and seems to be in agreement with the values proposed in literature [31], [32]. 


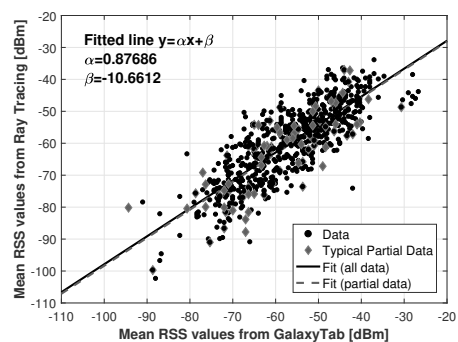

(a) Samsung Galaxy Tab

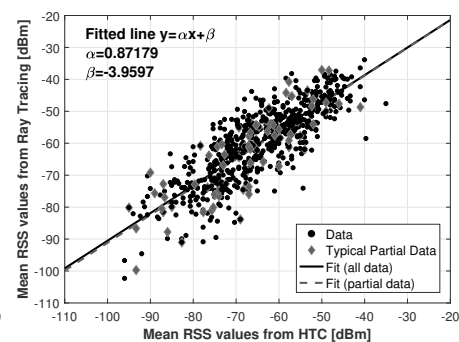

(b) HTC Desire HD

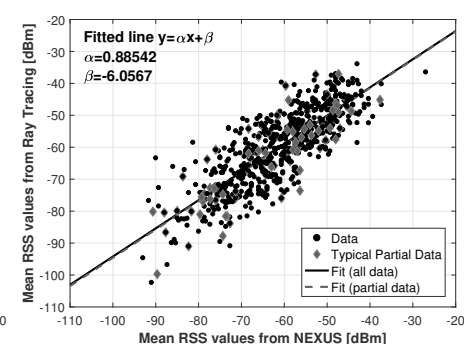

(c) Samsung Nexus $\mathrm{S}$

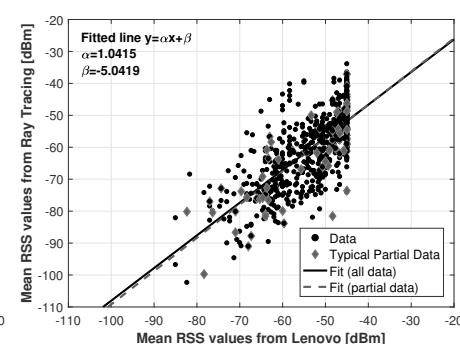

(d) Lenovo X100e

Fig. 3. Ray Tracing Radiomap calibration using data-fitting of the device-specific data

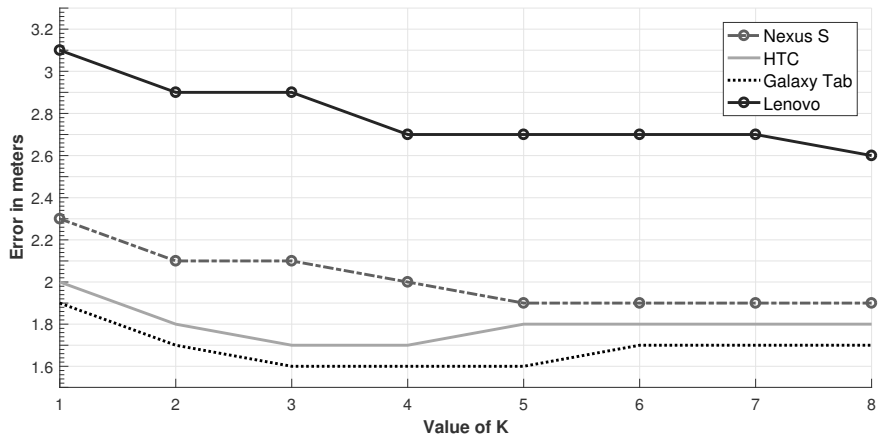

Fig. 4. Optimization of $K$ in the WKNN algorithm for the various devices

\section{A. No Device Calibration}

First, an examination of the performance when the RT radiomap is utilized without any calibration performed and the findings are summarized in Table [ The error performance in the case that manually collected, device-specific radiomaps are used, is shown in parentheses. Apart from the mean, median and maximum positioning error pertaining to the dataset, the $67^{\text {th }}$ and $95^{\text {th }}$ percentiles of the Cumulative Distribution Function (CDF) are also reported. It is observed that there is performance degradation of the positioning accuracy when a RT radiomap is used as opposed to device-specific radiomaps. This degradation seems to be worse for the Galaxy Tab and the Lenovo laptop since these two devices have very different reception characteristics (also the physical structure and dimensions are very different) than the HTC device, the measurements from which have been used to fine-tune the parameters of the RT model (see section IV-A). There seems to be similar degradation for the Nexus device compared to the HTC as these two devices appear to have very similar reception characteristics. Overall, the results suggest that there is room for improving the accuracy by means of data calibration.

TABLE I

POSITIONING ERROR [M] WITH A NON-CALIBRATEd RT RADIOMAP ${ }^{1}$

\begin{tabular}{lcccc}
\hline & Lenovo & HTC & Nexus & Galaxy Tab \\
\hline Mean & $4.2(2.7)$ & $2.1(1.7)$ & $2.4(2.0)$ & $2.3(1.6)$ \\
Median & $4.0(2.5)$ & $1.7(1.5)$ & $2.1(1.6)$ & $1.9(1.3)$ \\
$\mathbf{6 7 \%}$ cdf & $5.0(3.1)$ & $2.4(2.0)$ & $2.7(2.5)$ & $2.5(1.8)$ \\
$\mathbf{9 5 \%}$ cdf & $7.2(5.8)$ & $4.5(3.6)$ & $5.1(4.7)$ & $5.2(3.9)$ \\
Max & $10.9(11.0)$ & $11.2(9.8)$ & $14.5(11.0)$ & $9.0(6.3)$ \\
\hline
\end{tabular}

${ }^{1}$ The device-specific radiomaps performance is shown in brackets

\section{B. RT Radiomap Calibration for multiple devices}

The two linear transformation equation coefficients $(\alpha, \beta)$ extracted by the data fitting process described in section IV-B are used in a pre-processing step prior to the position estimation to calibrate (scale) the observed on-line measurement to better map to the fingerprints in the RT radiomap. The positioning accuracy associated with this process is shown in Table II (in brackets is the positioning error for the case where no linear transformation is applied). It becomes evident that this method improves the positioning accuracy; $0.9 \mathrm{~m}$ for the Lenovo device and $0.2-0.3 m$ for the Nexus and Galaxy devices respectively. There seems to be no improvement for the HTC device whose data has been used to fine tune the parameters of the RT model.

TABLE II

POSITIONING ERRor [M] WITH A CALIBRATED RT RADIOMAP ${ }^{1}$

\begin{tabular}{lcccc}
\hline & Lenovo & HTC & Nexus & Galaxy Tab \\
\hline Mean & $3.1(4.2)$ & $2.1(2.1)$ & $2.2(2.4)$ & $2.0(2.3)$ \\
Median & $2.7(4.0)$ & $1.8(1.7)$ & $1.9(2.1)$ & $1.8(1.9)$ \\
67\% cdf & $3.5(5.0)$ & $2.5(2.4)$ & $2.6(2.7)$ & $2.3(2.5)$ \\
95\% cdf & $6.4(7.2)$ & $4.6(4.5)$ & $4.5(5.1)$ & $4.3(5.2)$ \\
Max & $11.2(10.9)$ & $11.2(11.2)$ & $13.2(14.5)$ & $10.3(9.0)$ \\
\hline
\end{tabular}

${ }^{1}$ The non-calibrated RT radiomap performance is shown in brackets

As the requirement is to facilitate the construction of a device-independent fingerprint-based radiomap and the estimation of the calibration parameters relatively easily and quickly, the crucial question is how much fingerprint data needs to be collected using each device in order to estimate the linear transformation coefficients to ensure a good mapping between the RSSI values measured during positioning and the ones in the RT radiomap. In this experimental analysis the devicespecific training data is employed and the effect of using part of it for calibrating the RT radiomap for various different devices is studied, as detailed in Section IV-B In order to achieve a uniform random distribution of partial data, this data is elected by randomly selecting a number of fingerprints from each of the 7 regions defined in the scenario as described in Section $\amalg$ The investigation involves the study of the effect of selecting different number of fingerprint locations from each region. This random fingerprint selection per region was repeated 100 times and for each partial data size the average and standard deviation of the positioning error (mean and $95^{\text {th }}$ percentile) were estimated and are tabulated in Table III In 
this case all 30 RSSI samples available at each fingerprint location are used to calculate the mean RSSI fingerprints.

It seems that using the data from only 1-2 locations per region, provides the same performance with the case when using the data from all the 110 training locations. The standard deviation is also very low, indicating that the mean error is not affected by the selection of specific locations in each region. Likewise, the $95^{t h}$ error percentile which provides an insight about high errors anticipated during positioning, is also close to the case where all the training data is used. This demonstrates the effectiveness and efficiency of the partialdata device calibration process.

Another important issue is the number of samples, containing raw RSSI values, that need to be collected at each location in order to calculate the mean RSSI fingerprints and cancel out any possible fast fading effects. This is directly related to the time spent at a particular location for recording a sequence of samples. By varying the number of samples that contribute to the mean RSSI fingerprint at each location it was found that using only 5 samples per fingerprint location does not affect the performance of the proposed approach and provides the same positioning accuracy, as with the case of using all 30 samples; see Table IV. This means that very little time needs to be spent for collecting data and the device calibration overhead can be further reduced.

TABLE III

POSITIONING ERROR [M] USING A VARIABLE NUMBER OF TRAINING LOCATIONS PER REGION FOR DEVICE CALIBRATION

\begin{tabular}{lcccc}
\hline & Lenovo & HTC & Nexus & Galaxy Tab \\
\hline \multicolumn{4}{c}{ Mean error } \\
1 location & $3.1 \pm 0.2$ & $2.1 \pm 0.1$ & $2.2 \pm 0.2$ & $2.0 \pm 0.1$ \\
2 locations & $3.1 \pm 0.1$ & $2.1 \pm 0.0$ & $2.2 \pm 0.0$ & $2.0 \pm 0.0$ \\
All locations & 3.1 & 2.1 & 2.2 & 2.0 \\
\hline \hline & \multicolumn{4}{c}{$\mathbf{9 5 \%}$ CDF error } \\
1 location & $6.4 \pm 0.3$ & $4.6 \pm 0.2$ & $4.6 \pm 0.2$ & $4.5 \pm 0.2$ \\
2 locations & $6.4 \pm 0.1$ & $4.6 \pm 0.1$ & $4.5 \pm 0.1$ & $4.4 \pm 0.1$ \\
All locations & 6.4 & 4.6 & 4.5 & 4.3 \\
\hline
\end{tabular}

\section{Discussion}

Results indicate that for the Lenovo and Galaxy devices, the proposed approach improves considerably the performance compared to using the non-calibrated RT radiomap, while the positioning error comes closer to the one achieved when device-specific radiomaps are used. For the Nexus device similar behaviour was observed, while in the case of HTC the device calibration approach does not lead to significant improvements compared to the non-calibrated case since the RT radiomap was generated based on RT simulation parameters which have been optimised using measurements from the HTC device. The results indicate that the traditional fingerprintbased approach can be replaced by the proposed one. Here only 5 samples at 2 randomly selected fingerprint locations in each of the 7 regions inside the whole experimentation area for device calibration need to be collected. This translates into less than 5 minutes of data collection for each device, compared to around 2 hours of RSSI data logging for building each devicespecific radiomap. If we also consider that RT simulations for
TABLE IV

POSITIONING ERROR [M] USING 2 LOCATIONS PER REGION AND 5 SAMPLES PER LOCATION FOR DEVICE CALIBRATION ${ }^{1}$

\begin{tabular}{lcccc}
\hline & Lenovo & HTC & Nexus & Galaxy Tab \\
\hline \multirow{2}{*}{ Mean } & $3.1 \pm 0.1$ & $2.1 \pm 0.1$ & $2.3 \pm 0.1$ & $2.0 \pm 0.1$ \\
& $(2.7)$ & $(1.7)$ & $(2.0)$ & $(1.6)$ \\
Median & $2.7 \pm 0.1$ & $1.9 \pm 0.1$ & $2.0 \pm 0.0$ & $1.7 \pm 0.0$ \\
& $(2.5)$ & $(1.7)$ & $(1.6)$ & $(1.3)$ \\
67\% cdf & $3.5 \pm 0.1$ & $2.4 \pm 0.1$ & $2.6 \pm 0.1$ & $2.3 \pm 0.1$ \\
& $(3.1)$ & $(2.0)$ & $(2.7)$ & $(1.8)$ \\
95\% cdf & $6.5 \pm 0.2$ & $4.6 \pm 0.2$ & $4.6 \pm 0.2$ & $4.4 \pm 0.1$ \\
& $(5.8)$ & $(3.6)$ & $(4.7)$ & $(3.9)$ \\
Max & $11.1 \pm 0.1$ & $10.4 \pm 0.4$ & $12.8 \pm 0.6$ & $10.3 \pm 0.5$ \\
& $(11.0)$ & $(9.8)$ & $(11.0)$ & $(6.3)$ \\
\hline
\end{tabular}

${ }^{1}$ The device-specific radiomap performance is shown in brackets

this wireless environment took about 40 minutes to generate the radiomap the total time saving is around $60 \%$. Larger scale setups would lead to greater savings in time and labour.

\section{MAP-CONSTRAINED FINGERPRINTING}

\section{A. Route Probability Factor}

Although the approach presented above facilitates the easy creation and maintenance of device-independent fingerprinting radiomaps and achieves performance which approaches the one when using manually-collected device-specific radiomaps, the achieved accuracy is slightly above 2 meters. To fulfill the requirement for the sub-two-meter accuracy reported in [3] this work goes one step ahead and investigates potential accuracy improvements in the RT-based fingerprinting approach by imposing map constrains in the form of a-priori knowledge into the positioning methodology. This is based on the assumption that the movement of the users is expected to be constrained within specific regions and different probabilities could be assigned to different fingerprints reflecting the likelihood of the user being there. Therefore, this paper introduces the Route Probability Factor (RPF) which reflects these probabilities. This means that along a frequent route (an example is shown in Figure 5], the RPF will be increased, while in remote areas it will be decreased. The RPF does not only affect the probabilities along the specified route, but also the positions at its proximity. For this purpose, a normally distributed approach was implemented, at a radius $\rho$ across the route, creating route tubes. For every location on each frequent route, the algorithm assigns a decaying probability to all those fingerprints in the radiomap, which reside within a circle of radius $\rho$ around this location. This decaying probability is assumed to follow a Gaussian Normal Distribution which is a traditional method used in fingerprint database generation [44], [45] given by:

$$
R P F_{\ell_{i}}=R P F_{\ell}\left(\frac{1}{\sigma_{\text {route }} \sqrt{2 \pi}}\right) e^{-\frac{1}{2}\left(\frac{\left\|\bar{\ell}-\overline{\ell_{i}}\right\|}{\sigma_{\text {route }}}\right)^{2}}
$$

where $R P F_{\ell}$ is the Route Probability Factor at the location $\ell$ which lies exactly on the route and $\left\|\bar{\ell}-\overline{\ell_{i}}\right\|$ is the distance between location $\ell$ and any other location $\ell_{i}$ within the range of $\rho$. Finally, $\sigma_{\text {route }}$ is given with respect to the selected $\rho$ : for a $99 \%$ confidence level $\sigma_{\text {route }}=\rho / 3$, since statistically $3 \sigma$ provides this confidence level. 
This iterative process results in a normalised probability matrix which contains a probability $p\left(\ell_{i}\right)$ for every location along each frequent route tube. All these matrices are then summed up to result into an accumulated probability matrix which describes the likelihood of a user being in any location. This matrix has a one-to-one relation to the fingerprints database and is then used in conjunction with the positioning algorithm to improve the localisation accuracy.

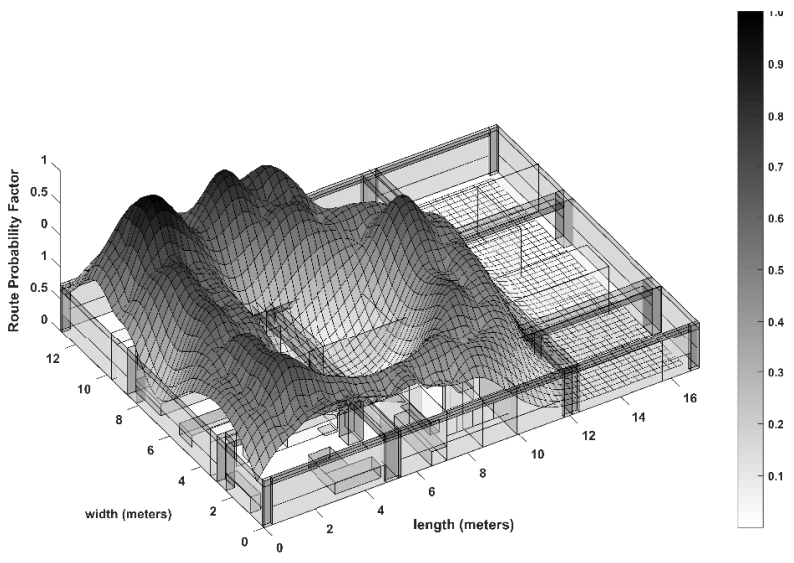

Fig. 5. Route Probability Factor - RPF

The same WKNN deterministic algorithm is used for positioning, extended with the probabilistic part of the RPF. The proposed approach takes into consideration the a-priori knowledge of the frequent user routes, as well as the weighted Euclidean distance of the observed location (see Eq. 2). The former allows to incorporate map constraints into the position estimation, by assigning the different likelihoods of each location in the environment, in the form of a probability matrix. In this context each fingerprint in the database is given a prior probability $p\left(\ell_{i}\right)=R P F_{i}$, which is used in Eq. (2). These prior probabilities can also be combined with the probabilities explicitly set to a minimum, in areas that are not accessible by the user. The normalised distribution of the RPF matrix used in this work is presented in Figure 5.

\section{B. Performance Evaluation}

The effectiveness of the RPF approach was evaluated with respect to the improvement in the positioning accuracy achieved when imposing map constrains through the extracted probability matrix which contains the probabilities $p\left(\ell_{i}\right)$ (see Eq. (2). The value of $K$ was, as previously, set to 4 .

The results when using the RPF approach are summarized in Table $\mathrm{V}$ for all the devices in consideration (in parentheses the non-RPF result is indicated) while the respective graphs showing the Cumulative Distribution Functions (CDF) of the obtained localisation accuracy are depicted in Figure 7

It is observed that when the map constraints are used, an improvement of around $20 \%$ occurs on the mean positioning accuracy for the Android devices whereas less improvement is observed for the laptop $(\sim 6 \%)$. There is also a significant reduction of $\sim 30 \%$ on the maximum error for the Android devices and $\sim 10 \%$ for the laptop. The improvement is sustained in the whole range of the error CDF, as it can be
TABLE V

POSITIONING ERROR [M] USING THE RPF APPROACH ${ }^{1}$

\begin{tabular}{lcccc}
\hline & Lenovo & HTC & Nexus & Galaxy Tab \\
\hline Mean & $2.9(3.1)$ & $1.7(2.1)$ & $1.9(2.3)$ & $1.6(2.0)$ \\
Median & $2.5(2.8)$ & $1.3(1.7)$ & $1.5(1.9)$ & $1.4(1.8)$ \\
67\% cdf & $3.5(3.6)$ & $1.9(2.5)$ & $2.5(2.6)$ & $1.9(2.3)$ \\
95\% cdf & $6.5(6.8)$ & $4.6(4.9)$ & $4.5(4.5)$ & $4.2(4.3)$ \\
Max & $10.9(11.2)$ & $7.4(10.2)$ & $10.9(13.2)$ & $7.7(10.3)$ \\
\hline
\end{tabular}

${ }^{1}$ Non-RPF resuls is shown in brackets.

seen in Figure 7 The position estimates for all points along the test route in the map-constrained scenario, are presented in Figure 6 with solid circle markers. It can be observed that, as a result of the integration of the RPF in the positioning procedure, the estimated positions were shifted from nonrealistic areas (cross and diamond markers) towards more reasonable positions, along or near the test route.

Given these accuracy improvements, it is interesting to investigate the effect of varying the distribution of the RPF as well as the route radius $\rho$ (see Eq. 3) around the frequent route used for the generation of the a-priori probability matrix. Similar work has been reported in [46]. In this context, different distributions and different values of $\rho$ for various resolutions of the fingerprint radiomap were investigated (resolution is defined as the spacing between fingerprints in the radiomap). Figure 8 indicates that the normal distribution used in this work performs equally well as the exponential distribution but outperforms the other distributions (linear, uniform and distance-ratio). Also, as it is illustrated in table VI which tabulates the mean and the $95^{\text {th }}$ percentile of the positioning error, the value of $\rho$ affects the localisation accuracy. The results indicate that the value of $\rho$, should be between 1 or 2 times the fingerprint radiomap resolution. This finding seems to be consistent for a wide range of values of $K$; Table VI tabulates the results for $K=4$ and $K=9$.

TABLE VI

EFFECT OF RPF RADIUS $\rho$ ON ACCURACY 1

\begin{tabular}{|c|c|c|c|}
\hline & $\rho=\mathbf{0 . 5 m}$ & $\rho=\mathbf{1 . 0 m}$ & $\rho=\mathbf{2 . 0 m}$ \\
\hline $\begin{array}{l}\text { Resolution }=1.00 \mathrm{~m} \\
\text { Resolution }=0.50 \mathrm{~m} \\
\text { Resolution }=0.25 \mathrm{~m} \\
\end{array}$ & $\begin{array}{l}1.70,4.70 \\
1.86,5.30 \\
\mathbf{1 . 9 7 , 5 . 5 3} \\
\end{array}$ & $\begin{array}{c}\mathbf{K}=\mathbf{4} \\
1.65,4.2 \\
\mathbf{1 . 8 6}, \mathbf{5 . 1 3} \\
2.07,5.78 \\
\end{array}$ & $\begin{array}{r}\mathbf{1 . 6 4}, \mathbf{4 . 2} \\
1.90,5.17 \\
2.36,6.85 \\
\end{array}$ \\
\hline $\begin{array}{l}\text { Resolution }=1.00 \mathrm{~m} \\
\text { Resolution }=0.50 \mathrm{~m} \\
\text { Resolution }=0.25 \mathrm{~m}\end{array}$ & $\begin{array}{l}1.62,4.34 \\
\mathbf{1 . 5 8}, \mathbf{3 . 9 0} \\
\mathbf{1 . 9 5}, \mathbf{5 . 5 7} \\
\end{array}$ & $\begin{array}{c}\mathbf{K}=\mathbf{9} \\
1.65,3.99 \\
1.78,5.10 \\
1.97,5.65\end{array}$ & $\begin{array}{c}\mathbf{1 . 5 5}, \mathbf{3 . 8 9} \\
1.84,5.13 \\
2.10,5.7 \\
\end{array}$ \\
\hline
\end{tabular}

\section{Performance in realistic device-handling scenarios}

The applicability and performance of the proposed approach has so far been demonstrated through measurements and simulations in which the user is assumed to have the same orientation while holding the device the same way at every location both during the off-line and the on-line phase. However, this rarely happens in practice and therefore it is important to 


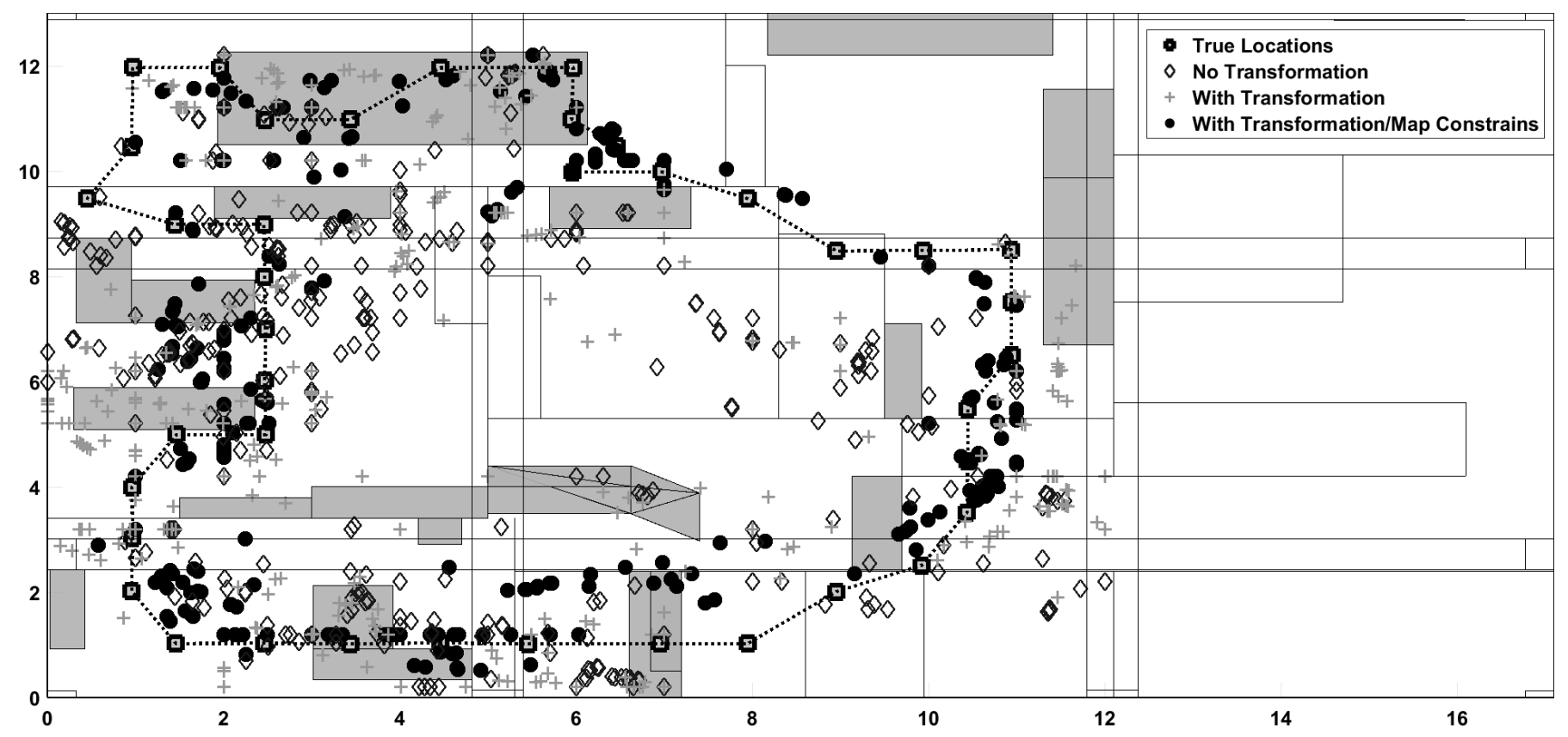

Fig. 6. Estimates along the test route

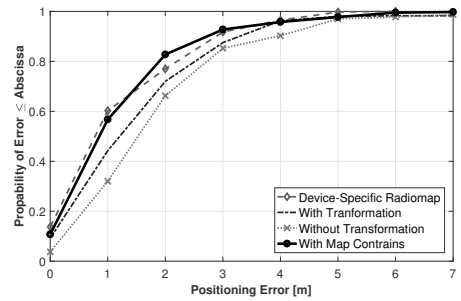

(a) Samsung Galaxy Tab

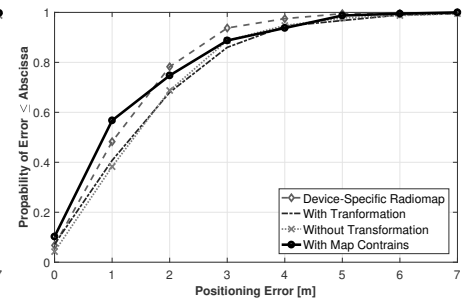

(b) HTC Desire HD

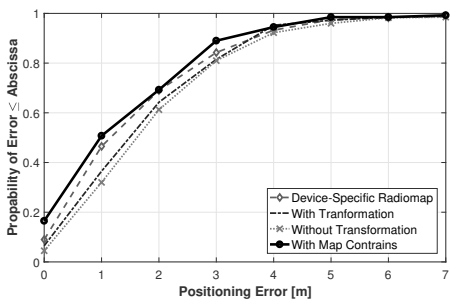

(c) Samsung Nexus S

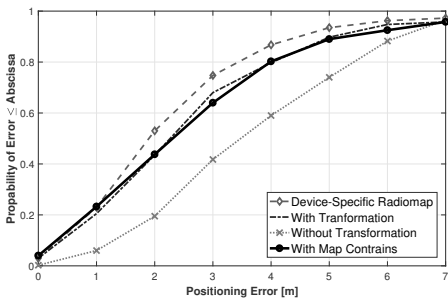

(d) Lenovo X100e

Fig. 7. CDF of Location Accuracy for the various devices

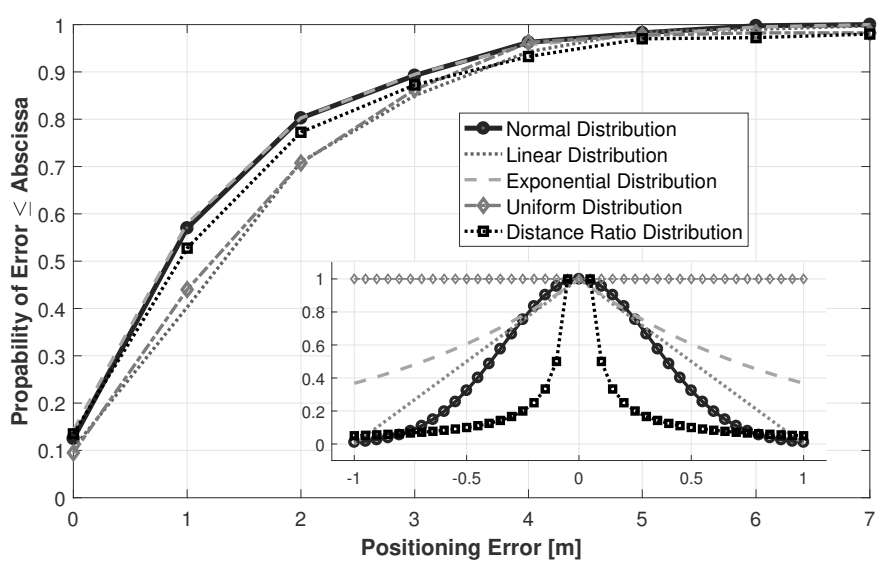

Fig. 8. Comparison of various RPF distributions

investigate how this approach would perform under realistic device-handling conditions which involve the impact of hands, the user orientation, the body blockage and the impact of keeping devices in a pocket. Therefore, additional test data were collected along the same test route as before with the user performing a random device-handling behaviour. The first investigation was carried out using the Galaxy Tab were at every test location 10 samples were collected with the user being randomly orientated while holding the tablet in different random ways (vertically, horizontally, etc.). As shown in Figure 9, this resulted in an RSSI variation (compared to the original test data) which follows a zero-mean normal distribution with a standard deviation of $4.2 \mathrm{~dB}$. These error statistics, which are inline with the findings reported in [47], resulted in an overall degradation of the positioning accuracy by roughly $0.5 \mathrm{~m}$ compared to the original case where the data were collected under specific orientations and specific devicehandling conditions (compare Tables $\mathrm{VII}$ and $\mathrm{V}$ ). However, as shown in Figure 11a the proposed RPF approach still improves the accuracy and makes it comparable to the case where device-specific radiomaps are used under these realistic conditions. It is also very common for the user to keep the smart-phone inside a pocket therefore additional test data were collected to accommodate this condition. Specifically, like before, 10 samples were collected at every location along the test route under different orientations and handling conditions but every $3^{\text {rd }}$ sample the smart phone was put in the pocket. The RSSI data collected experiences a variation compared to the original case, which, as shown in Figure 10, follows a 
bi-normal distribution. The first distribution is a zero-mean normal one with a standard deviation of $3.6 \mathrm{~dB}$ which is related to the different out-of-pocket handling conditions whereas the second one has a standard deviation of $5.1 d B$ and a mean of $6.3 d B$ which seems to be related to the additional attenuation of the signal due the fact that the device is in the pocket. As shown in Figure $11 \mathrm{~b}$ and Table VII there is again a consistent degradation of roughly $0.5 \mathrm{~m}$ in the positioning accuracy. Nevertheless, the RPF methods provide improvements, which, as before, approach the accuracy of the device-specific radiomaps case.

Similar performance has been observed when the same handling conditions were applied to the HTC Desire and the Lenovo Laptop and the results are shown in Figures $11 \mathrm{c}$ and 11d respectively and summarised in Table VII which demonstrate that even if there is a degradation in the accuracy due to the random device-handling behaviour of the user, the proposed approach makes the performance better.

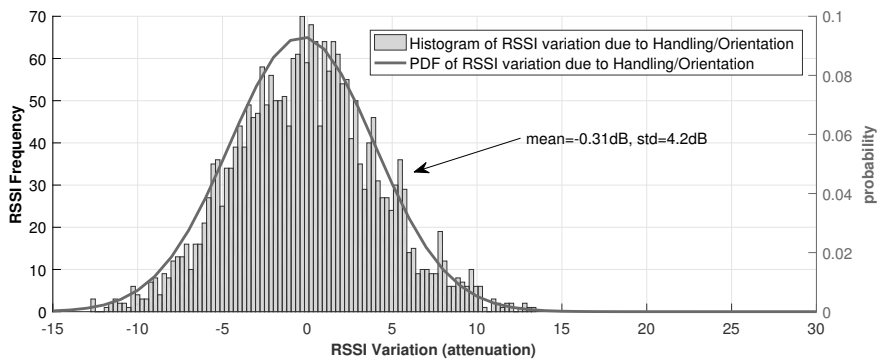

Fig. 9. Galaxy Tab RSSI variation under realistic device handling conditions
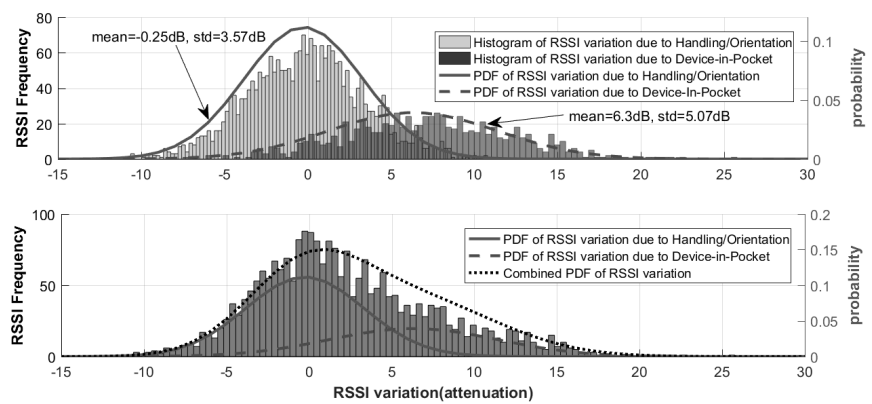

Fig. 10. Nexus RSSI variation under realistic device handling conditions

\section{Versatility of the RPF Approach}

It was shown that the RPF approach performs relatively well under realistic user-handling conditions. Although this could be a sign of versatility, it is also important to investigate the perfromance in a different environment and for different devices. In fact, the work presented in this paper is a continuation of the preliminary work reported in [27], the results of which are shown in Figure 12. In this work, a typical office environment was tested and the RPF method achieved an improvement of $28 \%$ in the mean positioning accuracy (from $2.03 \mathrm{~m}$ to $1.46 \mathrm{~m}$ ) and a radical reduction of $65 \%$ on the maximum error $(5.95 \mathrm{~m}$ instead of $17.09 \mathrm{~m})$. These results are in line with the ones reported in the current work. To
TABLE VII

POSITIONING ERROR [M] USING THE RPF APPROACH UNDER REALISTIC DEVICE-HANDLING CONDITIONS ${ }^{1}$

\begin{tabular}{lcccc}
\hline & Lenovo & HTC & Nexus & Galaxy Tab \\
\hline \multirow{2}{*}{ Mean } & 3.4 & 2.2 & 2.3 & 2.3 \\
& $(2.9,4.1)$ & $(2.1,2.8)$ & $(2.4,3.0)$ & $(2.1,2.6)$ \\
\multirow{2}{*}{ Median } & 2.9 & 1.6 & 1.9 & 1.7 \\
& $(2.5,3.9)$ & $(1.8,2.1)$ & $(1.9,2.5)$ & $(1.7,2.1)$ \\
\multirow{2}{*}{ 67\% cdf } & 4.2 & 2.6 & 2.7 & 2.5 \\
& $(3.3,4.8)$ & $(2.3,3.0)$ & $(2.7,3.4)$ & $(2.5,3.0)$ \\
95\% cdf & 7.8 & 5.8 & 5.3 & 6.4 \\
& $(5.9,7.4)$ & $(5.0,6.8)$ & $(6.0,6.5)$ & $(4.8,5.7)$ \\
\multirow{2}{*}{ Max } & 10.9 & 10.4 & 10.2 & 10.8 \\
& $(9.5,10.9)$ & $(11.6,13.6)$ & $(11.0,12.6)$ & $(9.4,11.1)$ \\
\hline
\end{tabular}

${ }^{1}$ The first number in the brackets is the error when device-specific radiomaps are used and the second is the error when neither calibration nor the RPF are used.

further demonstrate the versatility of the proposed method, test data were collected along the same test route as before using another four Android devices; Samsung GT-S7580, Samsung S8, HTC One M8 and LG G Pad 8.3. The results shown in Table VIII indicate that the RPF method performs equally well for a range of devices which proves its versatility.

TABLE VIII

POSITIONING ERROR [M] USING THE RPF APPROACH AND RT-GENERATED RADIOMAPS ${ }^{1}$

\begin{tabular}{lcccc}
\hline & GT-S7580 & HTC M8 & Samsung S8 & LG G Pad \\
\hline Mean & $1.9(2.8)$ & $1.9(3.1)$ & $1.9(3.2)$ & $1.9(2.5)$ \\
95\% cdf & $4.5(6.1)$ & $5.0(7.0)$ & $4.6(6.4)$ & $5.1(5.2)$ \\
Max & $10.9(15.4)$ & $10.3(14.9)$ & $10.9(14.4)$ & $9.9(8.5)$ \\
\hline
\end{tabular}

${ }^{1}$ Non-RPF resuls is shown in brackets.

\section{DISCUSSION}

Results indicate that the proposed methodology which combines radiomap generation using RT, data fitting and linear transformation and the RPF method could improve the mean positioning accuracy by $20-30 \%$ while reducing the maximum error by up to $35 \%$. This is comparable to various attempts reported in literature to improve the fingerprinting accuracy. For instance, the authors of [48] have theoretically demonstrated that the temporal correlation of the RSSI could be exploited to further improve fingerprinting accuracy by $13 \%$ $30 \%$. One of the advantages of the proposed method is its applicability when heterogeneous clients are used without the need for time-consuming exhaustive measurements using each device to populate the radiomap. This is done by using RT in combination with a calibration to fit the radiomap to the particular device. Kjrgaard in [19] used Hyperbolic Location Fingerprinting, which records fingerprints as signal strength ratios between pairs of transmitter and he reports a roomsize (assumed to be $4 m \times 4 m$ ) accuracy of around $50 \%$. The proposed method in this paper achieves an up-to-4 $m$ accuracy of around $90 \%$ for the Android devices and $80 \%$ for the laptop. However, the modern requirement in indoor positioning is in the sub-2-meter range [3] therefore a $4 m$ accuracy cannot be considered enough. The results in this paper indicate a mean error of around 1.6-1.9m could be achieved for the Android 


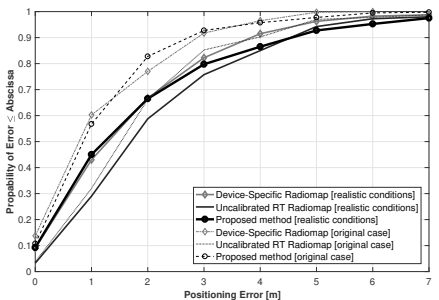

(a) Galaxy Tab

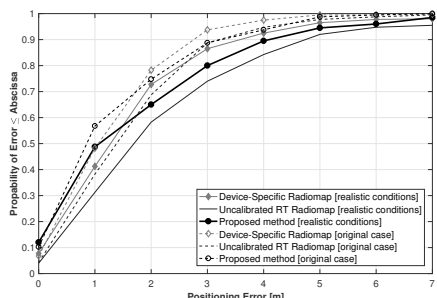

(b) HTC Desire HD

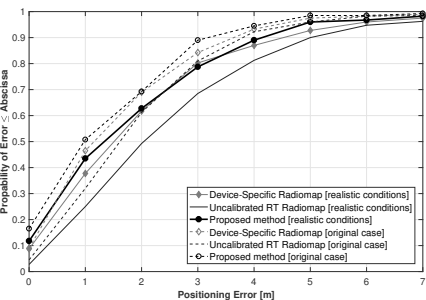

(c) Samsung Nexus $\mathrm{S}$

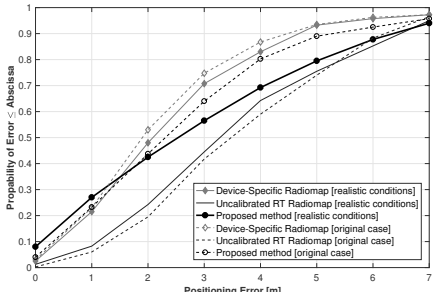

(d) Lenovo X100e

Fig. 11. Performance of the proposed approach under realistic device-handling conditions

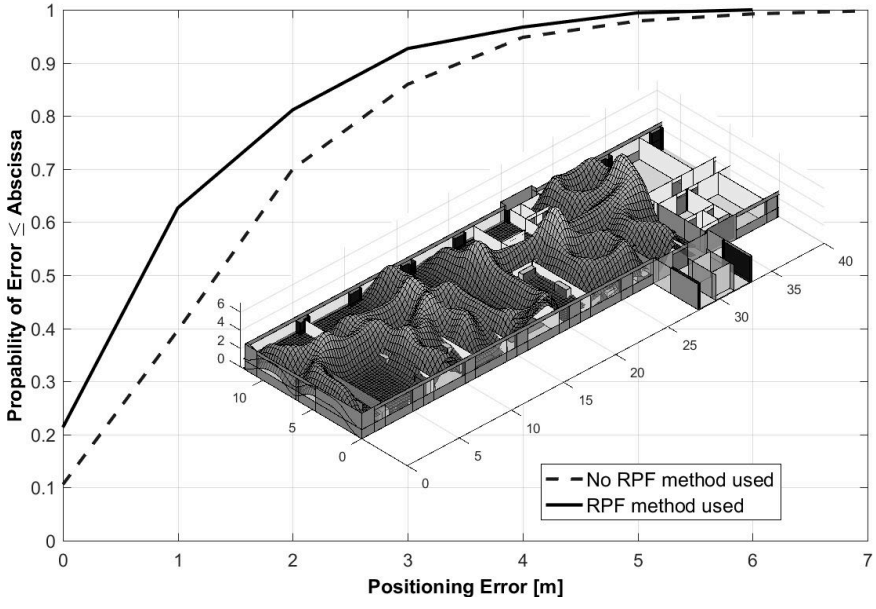

Fig. 12. PRF method in a different environment

devices which is better than the $2.44 m$ accuracy (LoS only) reported in [29] which also uses RT to generate the devicefree radiomap. Obviously, works have reported in literature which achieve fingerprinting accuracy in the sub-meter-range (e.g. UWB [4]-[6], MFI [7], [8]) however they require nonoff-the-shelf and usually expensive equipment.

\section{CONCLUSION}

Multi-device fingerprint-based positioning is an active research field because of the time-consuming data-collection process, using several different devices for the construction of the necessary radiomap. The focus in this work was on the use of a deterministic 3D Ray Tracing-based radio propagation model to generate a unique reference radiomap easily and quickly and subsequently to combine it with a device calibration process, based on linear data fitting, to better map the RSSI values observed during positioning to the reference radiomap irrespectively of the device used. The proposed approach mitigates the cumbersome task of recording large datasets of RSSI values throughout the area of interest using multiple devices. The performance evaluation indicated that only a small amount of device-specific data is required to reach the same level of positioning accuracy attained with a manually collected device-specific radiomap. The approach is far less laborious compared to traditional radiomap construction indicating around $60 \%$ saving in time as well as facilitating the possibility of easily re-generating the radiomap when something in the wireless environment is changed (e.g. APs added or removed, furniture relocated, etc.). This can be easily done by running the RT simulator, instead of collecting the radiomap data from scratch.

The introduction of weight coefficients in the form of apriori knowledge that takes the form of map constrains and reflects the likelihood of users being in particular locations can result in significant improvements in position estimation in indoor environments. In this direction this paper proposed the implementation of RPF as a matrix, which can be either populated manually, by observing the human mobility behaviour, or through the implementation of supervised or unsupervised learning methods. This could also be done on a personalizedlevel (assuming privacy issues are not raised) by keeping one probability matrix per individual user.

\section{REFERENCES}

[1] Y. Gu, A. Lo, and I. Niemegeers, "A survey of indoor positioning systems for wireless personal networks," IEEE Communications Surveys Tutorials, vol. 11, no. 1, pp. 13-32, First 2009.

[2] D. Sterling, G; Top, "Mapping the indoor marketing opportunity," Opus Research Report, 2014.

[3] IndoorAtlas, "A 2016 global research report on the indoor positioning market." The Rise of Indoor Positioning, 2016.

[4] M. Kok, J. D. Hol, and T. B. Schn, "Indoor positioning using ultrawideband and inertial measurements," IEEE Transactions on Vehicular Technology, vol. 64, no. 4, pp. 1293-1303, April 2015.

[5] G. D. Angelis, A. Moschitta, and P. Carbone, "Positioning techniques in indoor environments based on stochastic modeling of uwb round-triptime measurements," IEEE Transactions on Intelligent Transportation Systems, vol. 17, no. 8, pp. 2272-2281, Aug 2016.

[6] A. Cazzorla, G. D. Angelis, A. Moschitta, M. Dionigi, F. Alimenti, and P. Carbone, "A 5.6-ghz uwb position measurement system," IEEE Trans. on Instrum. and Meas., vol. 62, no. 3, pp. 675-683, March 2013.

[7] B. Kim and S. H. Kong, "A novel indoor positioning technique using magnetic fingerprint difference," IEEE Transactions on Instrumentation and Measurement, vol. 65, no. 9, pp. 2035-2045, Sept 2016.

[8] V. Pasku, A. D. Angelis, A. Moschitta, P. Carbone, J. O. Nilsson, S. Dwivedi, and P. Hndel, "A magnetic ranging-aided dead-reckoning positioning system for pedestrian applications," IEEE Trans. on Instrumentation and Measurement, vol. 66, no. 5, pp. 953-963, May 2017.

[9] P. Bahl and V. Padmanabhan, "RADAR: an in-building RF-based user location and tracking system," in IEEE International Conference on Computer Communications INFOCOM, vol. 2, 2000, pp. 775-784.

[10] M. Kjærgaard, "A taxonomy for radio location fingerprinting," in $3 r d$ international conference on Location-and context-awareness. SpringerVerlag, 2007, pp. 139-156.

[11] S. He and S. H. G. Chan, "Wi-fi fingerprint-based indoor positioning: Recent advances and comparisons," IEEE Communications Surveys Tutorials, vol. 18, no. 1, pp. 466-490, Firstquarter 2016.

[12] T. Deasy and W. Scanlon, "Simulation or measurement: The effect of radio map creation on indoor WLAN-Based localisation accuracy," Wireless Personal Communications, vol. 42, no. 4, pp. 563-573, 2007.

[13] A. Hatami and K. Pahlavan, "Comparative statistical analysis of indoor positioning using empirical data and indoor radio channel models," in 3rd IEEE Consumer Communications and Networking Conference (CCNC), vol. 2. IEEE, 2006, pp. 1018-1022. 
[14] K. El-Kafrawy, M. Youssef, A. El-Keyi, and A. Naguib, "Propagation modeling for accurate indoor WLAN RSS-based localization," in IEEE Vehicular Technology Conference Fall (VTC2010-Fall), 2010, pp. 1-5.

[15] V. Mohtashami and A. A. Shishegar, "Effects of geometrical uncertainties on ray tracing results for site-specific indoor propagation modeling," in 2013 IEEE-APS Topical Conference on Antennas and Propagation in Wireless Communications (APWC), Sept 2013, pp. 836-839.

[16] — " "Effects of inaccuracy of material permittivities on ray tracing results for site- specific indoor propagation modeling," in 2013 IEEEAPS Topical Conference on Antennas and Propagation in Wireless Communications (APWC), Sept 2013, pp. 1172-1175.

[17] A. Haeberlen, E. Flannery, A. M. Ladd, A. Rudys, D. S. Wallach, and L. E. Kavraki, "Practical robust localization over large-scale 802.11 wireless networks," in 10th international conference on Mobile computing and networking (MobiCom), 2004, pp. 70-84.

[18] J.-G. Park, D. Curtis, S. Teller, and J. Ledlie, "Implications of device diversity for organic localization," in Proc. of IEEE INFOCOM 2011.

[19] M. B. Kjærgaard, "Indoor location fingerprinting with heterogeneous clients," Pervasive and Mobile Computing, vol. 7, no. 1, pp. 31-43, 2011.

[20] M. Raspopoulos, B. Denis, M. Laaraiedh, J. Domnguez, L. de Celis, D. Slock, G. Agapiou, J. Stphan, and S. Stavrou, "Location-dependent information extraction for positioning," in 2012 International Conference on Localization and GNSS, June 2012, pp. 1-6.

[21] N. Paspallis and M. Raspopoulos, An Open Platform for Studying and Testing Context-Aware Indoor Positioning Algorithms. Cham: Springer International Publishing, 2017, pp. 39-50. [Online]. Available: http://dx.doi.org/10.1007/978-3-319-52593-8_3

[22] M. D'Souza, B. Schoots, and M. Ros, "Indoor position tracking using received signal strength-based fingerprint context aware partitioning,", IET Radar, Sonar Navigation, vol. 10, no. 8, pp. 1347-1355, 2016.

[23] P. Pivato, L. Palopoli, and D. Petri, "Accuracy of rss-based centroid localization algorithms in an indoor environment," IEEE Trans. on Instrum. and Measurement, vol. 60, no. 10, pp. 3451-3460, Oct 2011.

[24] P. H. Tseng, Y. C. Chan, Y. J. Lin, D. B. Lin, N. Wu, and T. M. Wang, "Ray-tracing-assisted fingerprinting based on channel impulse response measurement for indoor positioning," IEEE Trans. on Instrumentation and Measurement, vol. 66, no. 5, pp. 1032-1045, May 2017.

[25] D. Macii, A. Colombo, P. Pivato, and D. Fontanelli, "A data fusion technique for wireless ranging performance improvement," IEEE Trans. on Instrum. and Measurement, vol. 62, no. 1, pp. 27-37, Jan 2013.

[26] M. Raspopoulos, C. Laoudias, L. Kanaris, A. Kokkinis, C. Panayiotou, and S. Stavrou, "3d ray tracing for device-independent fingerprint-based positioning in wlans," in Positioning Navigation and Communication (WPNC), 2012 9th Workshop on, March, pp. 109-113.

[27] A. Kokkinis, M. Raspopoulos, L. Kanaris, A. Liotta, and S. Stavrou, "Map-aided fingerprint-based indoor positioning," in 2013 IEEE 24th Annual International Symposium on Personal, Indoor, and Mobile Radio Communications (PIMRC), Sept 2013, pp. 270-274.

[28] Y. Ji, S. Biaz, S. Pandey, and P. Agrawal, "ARIADNE: a dynamic indoor signal map construction and localization system," in 4th ACM Int'l Conf. on Mobile systems, applications and services, 2006, pp. 151-164.

[29] A. Eleryan, M. Elsabagh, and M. Youssef, "AROMA: Automatic generation of radio maps for localization systems," CoRR, vol. abs/1002.1834, 2010.

[30] V. Honkavirta, T. Perala, S. Ali-Loytty, and R. Piche, "A comparative survey of WLAN location fingerprinting methods," in 6th Workshop on Positioning, Navigation and Communication, 2009, pp. 243-251.

[31] R. Bahl and V. N. Padmanabhan, "Radar: An in building rf-based user location and tracking system," in INFOCOM, vol. 2, 2000, pp. 775-784.

[32] B. Li, J. Salter, A. Dempster, and C. Rizos, "Indoor positioning techniques based on wireless LAN," in 1st IEEE Intrnl. Conf. on Wireless Broadband and Ultra Wideband Communications, 2006, pp. 13-16.

[33] P. Bahl, V. Padmanabhan, and A. Balachandran, "Enhancements to the RADAR user location and tracking system," Microsoft Research, Tech. Rep. MSR-TR-00-12, February 2000.

[34] S. Saha, K. Chaudhuri, D. Sanghi, and P. Bhagwat, "Location determination of a mobile device using IEEE $802.11 \mathrm{~b}$ access point signals," in IEEE Wireless Communications and Networking, vol. 3, 2003, pp. 1987-1992.

[35] M. Youssef and A. Agrawala, "The Horus WLAN location determination system," in 3rd ACM International Conference on Mobile systems, applications, and services, 2005, pp. 205-218.

[36] T. Roos, P. Myllymaki, H. Tirri, P. Misikangas, and J. Sievanen, "A probabilistic approach to WLAN user location estimation," Int'l Jnl of Wireless Information Networks, vol. 9, no. 3, pp. 155-164, Jul. 2002.
[37] T. Vaupel, J. Seitz, F. Kiefer, S. Haimerl, and J. Thielecke, "Wi-Fi positioning: System considerations and device calibration," in Int'l Conf. on Indoor Positioning and Indoor Navigation (IPIN), 2010, pp. 1-7.

[38] L. Liao, D. Fox, J. Hightower, H. Kautz, and D. Schulz, "Voronoi tracking: location estimation using sparse and noisy sensor data," in Intelligent Robots and Systems, 2003. (IROS 2003). Proceedings. 2003 IEEE/RSJ International Conference on, vol. 1, 2003, pp. 723-728 vol.1.

[39] F. Evennou, F. Marx, and E. Novakov, "Map-aided indoor mobile positioning system using particle filter," in Proc. IEEE Wireless Communications and Networking Conf, vol. 4, 2005, pp. 2490-2494.

[40] F. N. Ltd. TruNET Wireless. http://www.fractalnetworx.com/

[41] R. G. Kouyoumjian and P. H. Pathak, "A uniform geometrical theory of diffraction for an edge in a perfectly conducting surface," Proceedings of the IEEE, vol. 62, no. 11, pp. 1448-1461, Nov 1974.

[42] S. Stavrou and S. Saunders, "Review of constitutive parameters of building materials," in 12th Intrnl. Conf. on Antennas and Propagation ICAP 2003, vol. 1. IET, 2003, pp. 211-215.

[43] J. Jemai, R. Piesiewicz, and T. Kurner, "Calibration of an indoor radio propagation prediction model at $2.4 \mathrm{GHz}$ by measurements of the IEEE $802.11 \mathrm{~b}$ preamble," in IEEE 61st Vehicular Technology Conference (VTC 2005-Spring), vol. 1, 2005, pp. 111-115.

[44] H. Hashemi, "The indoor radio propagation channel," Proceedings of the IEEE, vol. 81, no. 7, pp. 943-968, Jul 1993.

[45] L. Chen, B. Li, K. Zhao, C. Rizos, and Z. Zheng, "An improved algorithm to generate a wi-fi fingerprint database for indoor positioning," Sensors, vol. 13, no. 8, pp. $11085-11$ 096, 2013. [Online]. Available: http://www.mdpi.com/1424-8220/13/8/11085

[46] A. Kokkinis, L. Kanaris, M. Raspopoulos, A. Liotta, and S. Stavrou, "Optimizing route prior knowledge for map-aided fingerprint-based positioning systems," in The 8th European Conference on Antennas and Propagation (EuCAP 2014), April 2014, pp. 2141-2144.

[47] J. Luo and X. Zhan, "Characterization of smart phone received signal strength indication for wlan indoor positioning accuracy improvement," Journal of Networks, vol. 9, no. 3, pp. 739-746, Mar 2014.

[48] X. Tian, M. Wang, W. Li, B. Jiang, D. Xu, X. Wang, and J. Xu, "Improve accuracy of fingerprinting localization with temporal correlation of the rss," IEEE Trans. on Mobile Computing, vol. PP, no. 99, pp. 1-1, 2017.

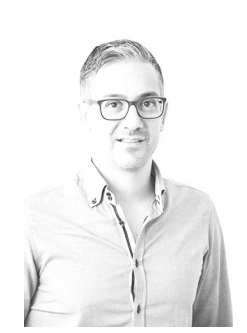

Marios Raspopoulos received an MEng in Electronics \& Mobile Communications (2003) and an MSc in Communications Networks \& Software (2004) from the University of Surrey, UK. From 2004 to 2008 he has been a Researcher at the Centre for Communications and Systems Research (CCSR) of the University of Surrey. In 2008 he has received his $\mathrm{PhD}$ in Telecommunication Engineering from the University of Surrey. He is currently an Assistant Professor in Electrical and Electronic Engineering at the University of Central Lancashire. His research interests span around the wide spectrum of ICT with special focus in Telecommunications and Mobile Wireless Communications \& Technologies with focus on Radio Propagation/Radio Planning, Electromagnetism, RF/Antenna Design and Wireless Channel Modelling, Localisation and Tracking, Information and Context-aware technologies, Ubiquitous and Pervasive computing, Cognitive Radio Technologies, Green and Energy Efficient Communication, Communication in Sensor Networks, Human Exposure to Electromagnetic Radiation etc. Dr Raspopoulos has participated in various indoor positioning-related multi-partner projects both in a national and an international level. 